UDC: 519.63

\title{
Application of mathematical fracture models to simulation of exploration seismology problems by the grid-characteristic method
}

\author{
M. V. Muratov ${ }^{1, a}$, I. B. Petrov ${ }^{1, b}$ \\ ${ }^{1}$ Moscow Institute of Physics and Technology, \\ 9 Institutskii per., Dolgoprudny, Russia \\ E-mail: ${ }^{\mathrm{a}}$ max.muratov@gmail.com, ${ }^{\mathrm{b}}$ petrov@mipt.ru
}

Received 22.05.2019.

Accepted for publication 14.11.2019.

In real problems of exploration seismology we deal with a heterogeneity of the nature of elastic waves interaction with the surface of a fracture by the propagation through it. The fracture is a complex heterogeneous structure. In some locations the surfaces of fractures are placed some distance apart and are separated by filling fluid or emptiness, in some places we can observe the gluing of surfaces, when under the action of pressure forces the fracture surfaces are closely adjoined to each other. In addition, fractures can be classified by the nature of saturation: fluid or gas. Obviously, for such a large variety in the structure of fractures, one cannot use only one model that satisfies all cases.

This article is concerned with description of developed mathematical fracture models which can be used for numerical solution of exploration seismology problems using the grid-characteristic method on unstructured triangular (in 2D-case) and tetrahedral (in 3D-case) meshes. The basis of the developed models is the concept of an infinitely thin fracture, whose aperture does not influence the wave processes in the fracture area. These fractures are represented by bound areas and contact boundaries with different conditions on contact and boundary surfaces. Such an approach significantly reduces the consumption of computer resources since there is no need to define the mesh inside the fracture. On the other side, it allows the fractures to be given discretely in the integration domain, therefore, one can observe qualitatively new effects, such as formation of diffractive waves and multiphase wave front due to multiple reflections between the surfaces of neighbor fractures, which cannot be observed by using effective fracture models actively used in computational seismology.

The computational modeling of seismic waves propagation through layers of mesofractures was produced using developed fracture models. The results were compared with the results of physical modeling in problems in the same statements.

Keywords: grid-characteristic method, exploration seismology problems, mathematical modeling, mathematical models of fractures, mesofractures, physical modeling

Citation: Computer Research and Modeling, 2019, vol. 11, no. 6, pp. 1077-1082.

This work was supported by the Russian Science Foundation, project no. 19-11-00023.

(C) 2019 Maksim V. Muratov, Igor B. Petrov This work is licensed under the Creative Commons Attribution-NoDerivs 3.0 Unported License. To view a copy of this license, visit http://creativecommons.org/licenses/by-nd/3.0/ or send a letter to Creative Commons, PO Box 1866, Mountain View, CA 94042, USA. 


\section{Introduction}

According to [Leviant et al., 2011], the geologists distinguish several types of fractures depending on dimensions: microfractures, whose aperture is about tens of microns and the extension is about several centimeters; mesofractures, whose aperture is about hundreds of microns and the extension is about several meters and macrofractures, whose aperture is about several millimeters and more, and the extension is from tens to hundreds of meters.

At the same time, for description of microfractures and mesofractures in the scale of geological medium regional exploration the use of popular effective models [Bakulin et al., 2004; Willis et al., 2006] will be the most optimal for performance. For modeling of mesofractures and macrofractures the modern high-performance computational systems allow us to use discrete models of fractures (Fig. 1), which significantly increase the possibilities of explorations.
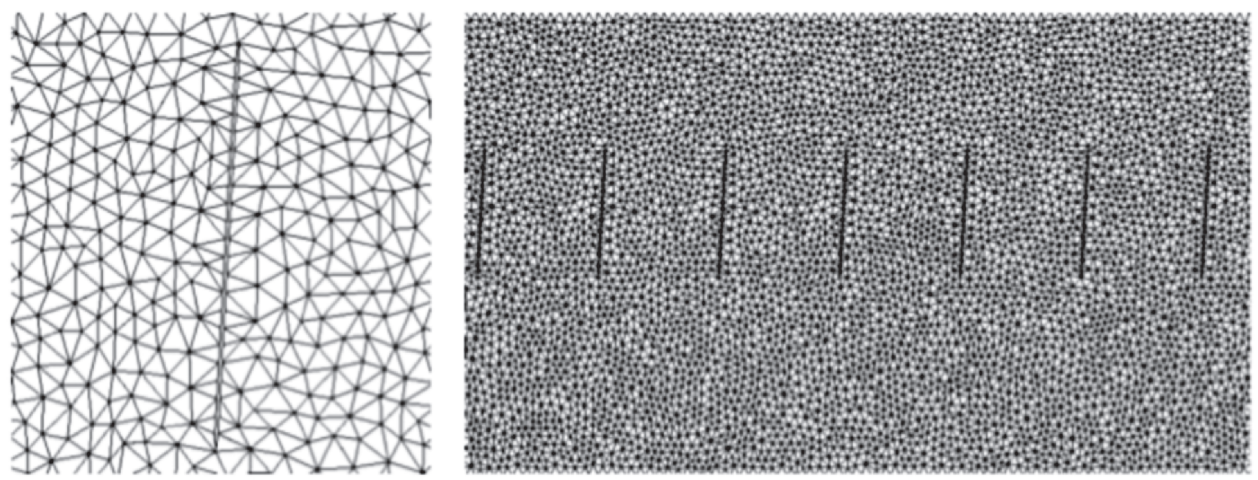

Figure 1. Examples of discrete models of fractures

For different problems one can use discrete models with different degrees of accuracy: fluid in fracture using the viscous liquid model, the model of an ideal fluid, the model with the state of elastic characteristics of liquid, etc. In the case of wave response observed in macroscale of the entire exploration seismology experiment the most optimal approach is to set fractures as boundary and contact boundary conditions - the model of an infinitely thin fracture. The applicability of this model was shown in [Leviant et al., 2012]. This article is devoted to selection of the most suitable conditions for different cases to solve exploration seismology problems using the grid-characteristic method [Magomedov, Kholodov, 1988; Petrov, Kholodov, 1984] on unstructured meshes [Aurenhammer, 1991; Edelsbrunner, 2006].

The cases most frequently used in practice are gas saturation, fluid filling and gluing of fracture. The range of the model was developed for these cases and their combinations.

\section{Mathematical model}

Wave processes in an elastic geological medium are described by equations of linear-elastic medium [Novatskii, 1975].

$$
\begin{aligned}
\rho \frac{\partial V}{\partial t} & =\frac{\partial \sigma_{j i}}{\partial x_{j}} \\
\frac{\partial \sigma_{i j}}{\partial t} & =\lambda\left(\sum_{k} \frac{\partial V_{k}}{\partial x_{k}}\right) I_{i j}+\mu\left(\frac{\partial V_{i}}{\partial x_{j}}+\frac{\partial V_{j}}{\partial x_{i}}\right)
\end{aligned}
$$

where $V_{i}$ is the velocity component, $\sigma_{i j}$ is the stress tensor component, $\rho$ is the density of medium, $\lambda$ and $\mu$ are Lame coefficients, and $I_{i j}$ is the component of the unit tensor. 
The 3D-system of such equations can be represented as

$$
\frac{\partial \vec{u}}{\partial t}+\sum_{i=1,2,3} \mathbf{A}_{\mathbf{i}} \frac{\partial \vec{u}}{\partial \xi_{i}}=0
$$

where $\xi_{i}$ takes values as $x, y$ and $z$, and $\vec{u}$ is the vector of values which can be represented as $\vec{u}=\left\{V_{x}, V_{y}, V_{z}, \sigma_{x x}, \sigma_{y y}, \sigma_{z z}, \sigma_{x y}, \sigma_{x z}, \sigma_{y z}\right\}$.

Such a system can be solved by the grid-characteristic method [Magomedov, Kholodov, 1988; Kulikovskii et al., 2001; Kholodov, 2008; Petrov, Lobanov, 2006].

\section{Fracture models}

This section is concerned with the developed models of fractures based on the model of an infinitely thin fracture. We consider the most popular cases such as gas-saturated, fluid-filled, glued and partially glued fractures. For each case a special model was developed.

\section{Gas-saturated (empty) fracture}

The model of gas-saturated (empty) fracture models well the behavior of fractures saturated by air or gas of a small depth up to $100-150 \mathrm{~m}$. At greater depths the fractures with air are closed by action of large pressure and gas acquires the properties of fluid.

The fracture can be set as the boundary condition of full reflection on the surfaces of the fracture:

$$
\mathbf{T} \vec{n}=0 .
$$

Such a model is applicable to describe the situation. The velocity of longitudinal elastic wave propagation in a geological medium $(1500$ to $7000 \mathrm{~m} / \mathrm{s})$ is much larger than the wave velocity in air $(330 \mathrm{~m} / \mathrm{s})$ and natural gas $(430 \mathrm{~m} / \mathrm{s})$ at small pressures. The velocity of shear elastic wave propagation in air is equal to zero. This is also the case for densities (1000 to $3000 \mathrm{~kg} / \mathrm{m}^{3}$ versus $1.2 \mathrm{~kg} / \mathrm{m}^{3}$ ). Therefore, the reflection coefficient is close to zero.

Thus, a fracture saturated by gas at pressures close to normal can be considered as empty and is set by the free boundary condition, which gives a full reflection of the falling wave.

\section{Fluid-filled fracture}

In most practical problems, fractures are filled by fluid: water, oil, liquefied gas, etc. So, it is expedient to develop a model allowing such a situation to be described.

Fluid-filled fracture is set as contact boundary with the free slide condition:

$$
\vec{v}_{a} \cdot \vec{n}=\vec{v}_{b} \cdot \vec{n}, \quad \vec{f}_{n}^{a}=\vec{f}_{n}^{b}, \quad \vec{f}_{\tau}^{a}=\vec{f}_{\tau}^{b}=0 .
$$

Such a contact boundary fully passes any longitudinal vibrations without reflection and fully reflects shear waves. This situation corresponds to a real one: the values of longitudinal waves propagation in fluid velocities and densities are comparable with velocities and densities of real geological media. At the same time, the velocities of shear vibrations in fluids are close to zero.

\section{Glued fracture}

At great depths, under the action of pressure the surfaces of fracture can be closed as elastic waves almost fully propagating through the fracture. In such cases it is best to use the contact condition of gluing:

$$
\vec{v}_{a}=\vec{v}_{b}, \vec{f}_{a}=-\vec{f}_{b},
$$

where $\vec{v}$ are the velocities of closing boundary points, $\vec{f}$ are the forces acting on the boundary, " $a$ " is the first and " $b$ " is the second surface of fracture. 


\section{Partially glued fracture}

In real exploration seismology we deal mostly with partially gluing fractures, where part of lateral surfaces is gluing and the other part is separated by fluid or gas. Such fractures give a partial propagation elastic wave front, which influences the response wave amplitudes of seismograms.

A model of fracture has been developed. In this model, at different points of surfaces, conditions for gas saturation (fluid filling) and full gluing are set randomly. The number of points of each type is regulated by the weight coefficient - the coefficient of gluing. Such a model enables us to set gas-saturated and fluid-filling fractures with fractures of gluing points from 0 to $100 \%$.

As the fracture reflects the wave front at some points and propagates it at other points, the superposition of diffracted waves formed by wave interaction with all points is the response of a gassaturated (fluid-filled) fracture with a smaller amplitude.

\section{Results of modeling}

We produce the numerical modeling of wave response from the layer of mesofractures. The results obtained were verified by methods of physical modeling. In modeling we used a geological medium with elastic characteristics $V_{p}=2260 \mathrm{~m} / \mathrm{s}, V_{s}=1300 \mathrm{~m} / \mathrm{s}, \rho=1200 \mathrm{~kg} / \mathrm{m}^{3}$. In a medium at a depth of $1300 \mathrm{~m}$ there was a layer of mesofractures. Their height was $12 \mathrm{~m}$, the angle was $50^{\circ}$, and the distance between fractures was about $12 \mathrm{~m}$. There were several rows of fractures, the common thickness of the layer was $160 \mathrm{~m}$. The horizontal dimension of the layer was $3000 \mathrm{~m}$. The initial signal is the spherical wave of explosion. The observation was produced on the horizontal line of seismoreceivers on the surface and in a vertical well using the VPS method. The scheme of experiment is represented in Fig. 2.
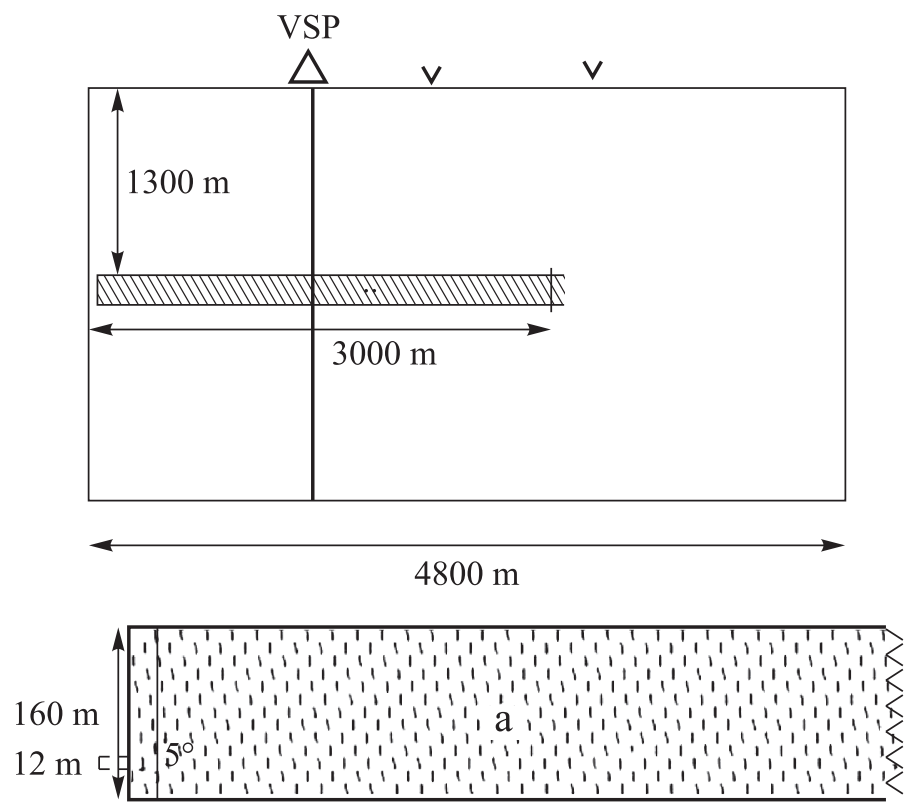

Figure 2. Examples of discrete models of fractures

A synthetical seismogram of the vertical component of the response obtained from the horizontal line of receivers on the surface by mathematical modeling is represented in Fig. 3a. Figure 3b shows the results of physical modeling for the same case. We can observe a good conformity.

The results of modeling in the case of VSP-observation are shown in Fig. 4. Figures $4 \mathrm{a}$ and $4 \mathrm{~b}$ show the results of mathematical and physical modeling, respectively. 


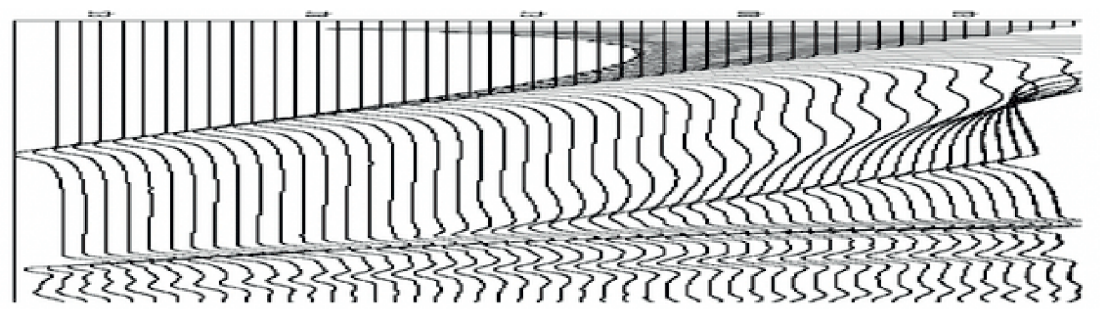

(a)

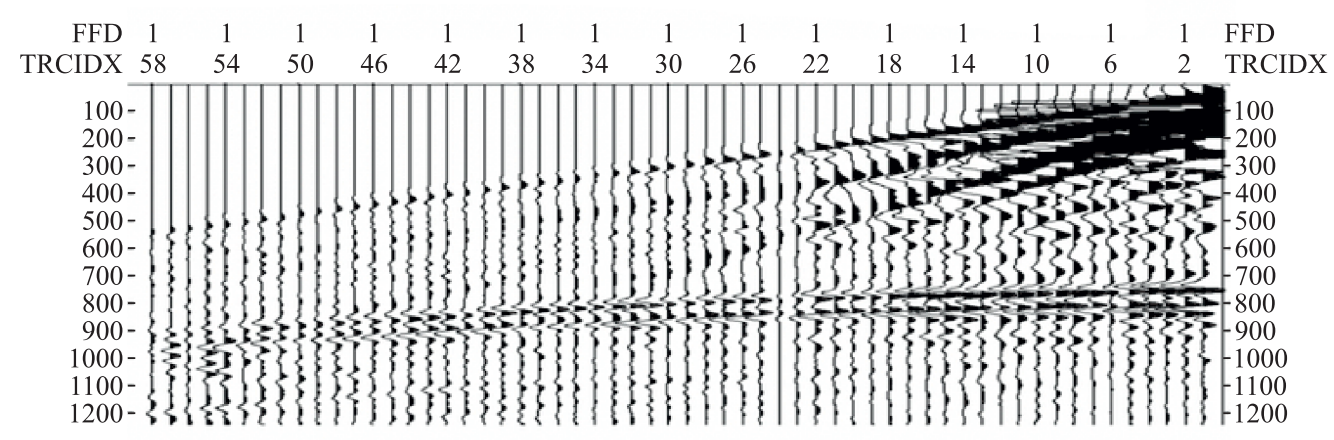

(b)

Figure 3. Examples of discrete models of fractures

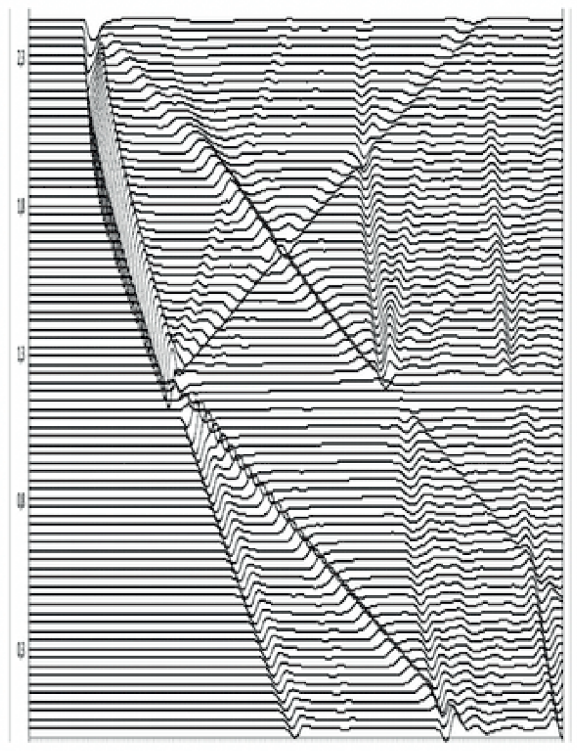

(a)

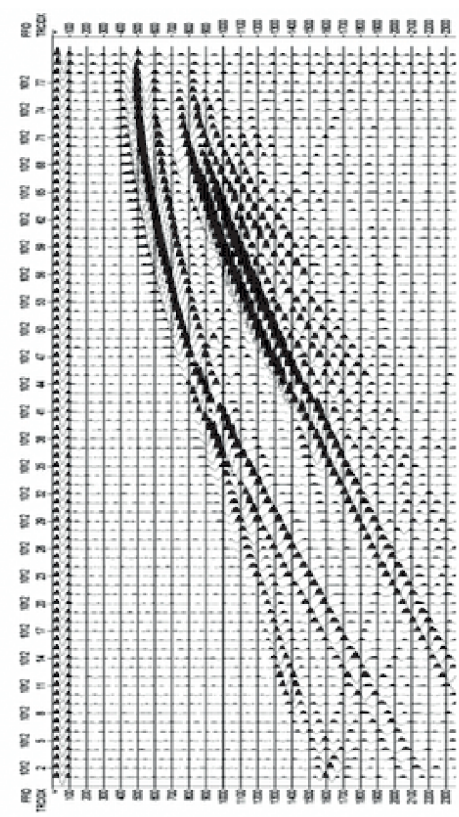

(b)

Figure 4. Examples of discrete models of fractures

\section{Conclusions}

In conclusion it worth to note that the absolutely thin fracture is a good model to simulate the behavior of response waves in exploration seismology problems. The computations using it do not require so much computational resources and their correctness is confirmed by methods of physical modeling. 


\section{References}

Aurenhammer F. Voronoi diagrams - a study of fundamental geometric data structure // ACM Computer Surveys. - 1991. - No. 23. - P. 345-405.

Bakulin A., Grechka V., Karaev N., Anisimov A., Kozlov E. Physical modeling and theoretical studies of seismic reflections from a fault zone // SEG. - 2004.. - P. 1674-1677.

Biryukov V.A., Muratov M.V., Petrov I. B., Sannikov A.V., Favorskaya A. V. Application of the gridcharacteristic method on unstructured tetrahedral meshes to the solution of direct problems in seismic exploration of fractured layers // Computational Mathematics and Mathematical Physics. 2015. - Vol. 55, No. 10. - P. 1733-1742.

Edelsbrunner $H$. Geometry and topology for mesh generation. - Cambridge: Cambridge University Press, 2006. $-177 \mathrm{p}$.

Kholodov A.S. Chislennye metody resheniya uravnenii i sistem giperbolicheskogo tipa [Numerical methods of hyperbolic equations and equations systems solution] // Enciklopedia nizkotemperaturnoi plazmy (seria "B”). - Moscow: Yanus-k, 2008. - Vol. VII-1, Part 2. - P. 91-103 (in Russian).

Kulikovskii A. G., Pogorelov N.V., Semeniv A. Yu. Matematicheskie voprosy chislennogo resheniya giperbolich-eskikh sistem uravnenii [Mathematical aspects of hyperbolic equation systems numerical solution]. - Moscow: FIZMATLIT, 2001. - 608 p. (in Russian).

Leviant V.B., Petrov I.B., Kvasov I.E. Numerical modeling of seismic response from subvertical macrofractures as possible fluid conduits. - 2011. - No. 4. - P. 41-61.

Leviant V.B., Petrov I. B., Muratov M.V. Numerical simulation of wave responses from subvertical macrofractures system // Seismic Technologies. - 2012. - No. 1. - P. 5-21.

Magomedov K. M., Kholodov A.S. Setochno-kharakteristicheskie chislennye metody [Grid-characteristic numerical methods]. - Moscow: Nauka, 1988. - 290 p. (in Russian).

Novatskii V. K. Teoriya uprugosti [Elasticity theory]. - Moscow: Mir, 1975. - 872 p. (in Russian).

Petrov I. B., Kholodov A.S. Numerical study of some dynamic problems of the mechanics of a deformable rigid body by the mesh-characteristic method // Computational Mathematics and Mathematical Physics. - 1984. - Vol. 24, No. 3. - P. 61-73.

Petrov I. B., Lobanov A.I. Lektsii po vychislitelnoi matematike [Computational mathematics lectures]. - Moscow: Internet-University of Informational Technologies, 2006. - 523 p. (in Russian).

Willis M.E., Burns D. R., Rao R., Minsley B., Toksoez M. N., Vetri L. Spatial orientation and distribution of reservoir fractures from scattered seismic energy // Geophysics. - 2006. - Vol. 71, No. 5. P. O43-O51. 